\title{
Análisis de patrones en asaltos de espada de alto nivel
}

\author{
Analysis of patterns in bouts elite epee
}

\section{Análise dos padrôes em assaltos espada elite}

\author{
Rafael Tarragó ${ }^{1}$, Xavier Iglesias ${ }^{2 *}$, Juan José Michavila ${ }^{3}$, Diego Chaverri ${ }^{2}$, Laura Ruiz-Sanchis ${ }^{4}$ y M. Teresa Anguera ${ }^{5}$ \\ ${ }^{1}$ Grup d'Investigació Social i Educativa de l'Activitat Física i l'Esport, INEFC-Universitat de Barcelona, ${ }^{2}$ INEFC-Barcelona Sport Sciences Research Group, Institut \\ Nacional d'Educació Física de Catalunya, INEFC-Universitat de Barcelona, ${ }^{3}$ Federació Catalana d'Esgrima, ${ }^{4}$ Facultad de Ciencias de la Actividad Fisica, Universidad \\ Católica de Valencia "San Vicente Mártir", ${ }^{5}$ Departamento de Metodologia de las Ciencias del Comportamiento, Facultad de Psicologia, Universidad de Barcelona
}

Resumen: El objetivo de este estudio fue la caracterización técnico-táctica de las acciones de combate en asaltos de esgrima de competición, determinando su eficacia en función del período del asalto en que se realizan, analizando el tiempo efectivo de combate y de pausa, e identificando si existen regularidades en las conductas de los esgrimistas en competición. Para ello se utilizó un diseño observacional puntual, nomotético y multidimensional. El instrumento observacional ESGRIMOBS fue validado mediante un panel de expertos compuesto por 17 especialistas. Se utilizó como instrumento de registro el programa LINCE, y los registros obtenidos fueron tratados y recodificados a través del programa Microsoft $^{\circ} \mathrm{Office}$ Excel $^{\triangleright}{ }^{\circ}$ 2013. El análisis estadístico descriptivo e inferencial se realizó mediante el programa PASW Statistics para Windows y el análisis de patrones a través de Theme. La fiabilidad en la observación se determinó mediante el grado de concordancia intra e interobservador. Se analizaron 14 asaltos del Campeonato del Mundo de espada masculina de categoría absoluta. El 43,1 \% $( \pm 13,3)$ de las acciones analizadas fueron eficaces, apreciándose un claro incremento de la eficacia en el transcurso del asalto. A medida que avanza el asalto el tiempo efectivo de combate disminuye y se alargan los tiempos de pausa. Se identificaron 72 T-Patterns que mostraron distintas regularidades en los 14 combates analizados. En conclusión, se dispone de una herramienta válida para el análisis técnico-táctico y de la estructura temporal de asaltos de esgrima, pudiendo configurarse la detección de T-Patterns como una nueva estrategia cualitativa en el análisis táctico de la esgrima.

Palabras clave: Esgrima, caracterización técnico-táctica, eficacia, T-Pattern, metodología observacional.

Abstract: The present study intended to characterize the tactical and the technical fighting actions during fencing competition bouts, determining the respective efficacy regarding the period that actions tended to occur. For that, the time of pause and the time of effective fighting, as well as the regularities found on fencers' behaviour were analysed. A punctual, nomothetic and multidimensional study design was used. ESGRIMOBS observational tool, validated by 17 experts, and LINCE recording instrument were applied. Data was treated and recodify through Microsoft $t^{\circ} O f-$ fice Excel $^{\circ}$ 2013. Data descriptive and inferential analyses were performed with the use of PASW Statistics for Windows, and T-Patterns detection was made by Theme software. Reliability was determined through intra and inter-observer agreement. 14 bouts of male epee World Cup of the absolute category were analysed. We verified that $43,1 \%( \pm 13,3)$ of the analysed actions proved effective; consequently an increase of the efficacy all over the bout was found. Also, during the bout, its effective time decreased and, contrarily, increased the pause periods. 72 T-Patterns were identified, showing different regularities in the 14 studied bouts. We conclude that tools applied in the present study are valid to perform tactical and technical analysis, as well as to identify temporal structures in fencing rounds. Thus, T-Patterns detection can be performed with the above-referred instruments, being a new qualitative strategy to analyse tactics in fencing sport.

Key words: Fencing, technical and tactical characterization, efficacy, $T$ Pattern, observational methodology.

Resumo: $\mathrm{O}$ objetivo do presente estudo foi a caracterizaçâo técnico-tática das açôes de combate em assaltos de esgrima de competiçấo, determinando-se a sua eficácia em função do período em que se realizam. Para isso, analisou-se o tempo de pausa e de combate efetivo, bem como se indagaram as regularidades existentes nas condutas dos esgrimistas de competiçấo. Utilizou-se um desenho observacional pontual, nomotético e multidimensional. O instrumento de observação utilizado designa-se por ESGRIMOBS, tendo sido validado por um painel composto por 17 especialistas, enquanto que o instrumento de registo foi o programa LINCE. Os dados recolhidos foram tratados e recodificados através do programa Microsoft $t^{\circ}$ Office Excel ${ }^{\circledR} 2013$. A análise descritiva e inferencial dos dados realizou-se com o recurso ao software PASW Statistics para Windows, enquanto que a análise de padrōes temporais efetuou-se através do software Theme. A fiabilidade dos dados foi determinada através do grau de concordância intra e inter-observadores. Analisaram-se 14 assaltos do Campeonato do Mundo de espada masculina de categoria absoluta. Verificou-se que 43,1\% $( \pm 13,3)$ das açóes analisadas revelaram-se eficazes, compreendendo-se portanto um claro aumento da eficácia no decurso do assalto. À medida que o combate decorre, o tempo efetivo do assalto diminui e aumentam os tempos de pausa. Identificaram-se 72 T-Patterns, que mostraram distintas regularidades nos 14 combates analisados. Conclui-se que as ferramentas utilizadas no presente estudo são válidas para a análise técnico-tática e das estruturas temporais em assaltos de esgrima, podendo assim configurar-se a deteçáo de T-patterns como uma nova estratégia qualitativa para a análise tática em esgrima.

Palavras chave: Esgrima, caracterização técnico-tática, eficácia, T-Pattern, metodologia observacional.

\section{Introducción}

La esgrima es un deporte de combate que forma parte del

Dirección para correspondencia [Correspodence address]: Xavier Iglesias. INEFC-Barcelona Sport Sciences Research Group. Institut Nacional d`Educació Física de Catalunya. Avda. de l’Estadi, 12-22 (Anella Olímpica de Montjuïc). 08038 Barcelona (Spain). E-mail: xiglesias@gmail.com programa olímpico desde la primera edición de los Juegos de la era moderna, en el que dos competidores (esgrimistas o tiradores) se enfrentan, con el objetivo de tocar a su rival y no ser tocados. Existen tres modalidades de armas diferentes, espada, florete y sable, que comparten algunas normas 
en común, pero también tienen sus propias particularidades, como la zona del cuerpo en que se considera que el tocado es válido (FIE, 2014).

Diferentes autores han apuntado que la literatura científica en esgrima no es abundante (Aquili, et al., 2013; Roi y Bianchedi, 2008; Turner, et al., 2013), aunque en los últimos ańos se han publicado interesantes trabajos relacionados con temáticas muy dispares, como la valoración funcional de los esgrimistas (Bressan y Ranzani, 1998; Iglesias, 1997; Lavoie, Léger y Marini, 1984; Tsolakis y Vagenas, 2010), las patologías y lesiones (Bonifazi, Rossi y Vannoni, 2009; Naghavi, 2002; Roi y Fasci, 1988), el tiempo de reacción y el sentido del tiempo (Gutiérrez-Dávila, Zingsem, Gutiérrez-Cruz, Giles y Rojas, 2014; Harmenberg, Ceci, Barvestad, Hjerpe y Nyström, 1991; Williams y Walmsley, 2000) o la biomecánica (Gholipour, Tabrizi y Farahmand, 2008; Lin, 2004).

La estructura de los asaltos, con el análisis de tiempos de actuación y pausa en competición, ha sido estudiada en diferentes niveles de esgrimistas (Aquili, et al., 2013; Iglesias, 1997; Lavoie, Léger, Pitre y Marini, 1985; Wylde, Tan y O’Donoghue, 2013), pero son escasas las referencias que describan la tipología de las acciones y la eficacia de las situaciones de combate (Iglesias, González, Cortés, Tarragó y García, 2008). Iglesias, Gasset, González y Anguera (2010) valoraron en espadistas de alto nivel las acciones más eficaces en función del contexto del combate, y detectaron patrones derivados de conductas catalizadoras en acciones en competición, a través de un análisis secuencial de retardos. La técnica analítica de detección de patrones temporales, denominados T-Patterns (Magnusson, 1996, 2000, 2005, 2006), se ha ido incorporando progresivamente al análisis de las regularidades en los deportes (Borrie, Jonsson y Magnusson, 2002), estableciendo relaciones significativas entre las conductas deportivas y sus estructuras temporales y secuenciales. Esta técnica de análisis no ha sido aplicada todavía en la esgrima, pero sí en otros deportes de combate (Camerino, Prieto, Lapresa, Gutiérrez-Santiago y Hileno, 2014; Gutierrez-Santiago, Prieto, Camerino y Anguera, 2011; Lapresa, Ibáñez, Arana, Garzón y Amatria, 2011) y de colectivos y de equipo (Camerino, Chaverri, Anguera y Jonsson, 2012; Fernández, Camerino, Anguera y Jonsson, 2009; Jonsson, et al., 2006; Lapresa, García, Arana y Garzón, 2011).

En este estudio se presenta un nuevo instrumento de observación de la esgrima, con el objetivo de lograr la caracterización técnico-táctica de las acciones de combate en asaltos de espada, determinando su eficacia en función del período del asalto, analizando su estructura temporal e identificando T-Patterns en las interacciones entre esgrimistas.

\section{Método}

Diseño

El diseño observacional utilizado en este estudio (Anguera, Blanco-Villaseñor, Hernández-Mendo y Losada, 2011) fue N/P/M (Nomotético/Puntual/Multidimensional), nomotético, por el análisis diferencial de los tiradores, puntual, al tratarse del registro en una única competición y sin seguimiento, y multidimensional, puesto que las dimensiones se corresponden con los distintos criterios del instrumento observacional.

\section{Participantes}

Se realizó la observación de los dieciséis tiradores de espada que accedieron a los octavos de final del campeonato del Mundo de espada de categoría absoluta disputado en Budapest el ańo 2013. Los deportistas $(n=16)$ fueron analizados en catorce asaltos $(n=14)$ : los octavos de final $(n=8)$, los cuartos de final $(n=4)$, una semifinal $(n=1)$ y la final $(n=1)$. El estudio se realizó de acuerdo a los requisitos establecidos en la declaración de Helsinki (Harriss y Atkinson, 2011) y fue sometido y aprobado por el comité ético de investigación clínica del deporte de Cataluña.

\section{Instrumentos}

\section{Instrumento observacional}

Se construyó un instrumento observacional ad hoc mediante el cual se podía lograr la caracterización técnico-táctica de las acciones de esgrima. El instrumento, denominado ESGRIMOBS, se diseñó para ser compatible con el análisis de las tres modalidades de la esgrima (espada, florete y sable). En la tabla 1 quedan descritas las distintas categorías en que quedaron divididos los 6 criterios del análisis multidimensional utilizados para este trabajo (sólo se utilizaron 6 de los 68 criterios que conforman ESGRIMOBS). 
Tabla 1. Criterios y categorías del instrumento de observación.

\begin{tabular}{|c|c|c|}
\hline Criterios & Categorias & Descripción \\
\hline \multirow{5}{*}{ PERIODO } & IPER & Primer periodo del asalto \\
\hline & 2PER & Segundo periodo del asalto \\
\hline & 3PER & Tercer periodo del asalto \\
\hline & PRORR_FT & Prórroga de 1 minuto por finalización del tiempo reglamentario \\
\hline & PRORR_NC & Prórroga de 1 minuto por no combatividad en el tercer período \\
\hline \multirow{3}{*}{ PRESIÓN } & PRES_I & Presión evidente del tirador de la izquierda de la imagen \\
\hline & PRES_D & Presión evidente del tirador de la derecha de la imagen \\
\hline & NPRES & No existe una presión evidente de ninguno de los dos tiradores \\
\hline \multirow{3}{*}{ PREPARACIÓN } & PREP_I & Preparación última del tirador de la izquierda \\
\hline & PREP_D & Preparación última del tirador de la derecha \\
\hline & NPREP & No hay preparación última de ninguno de los dos tiradores \\
\hline \multirow{6}{*}{ ACCIÓN INICIAL } & INICI_OF_I & Acción inicial ofensiva del tirador de la izquierda \\
\hline & INICI_OF_D & Acción inicial ofensiva del tirador de la derecha \\
\hline & INICI_DEF_I & Acción inicial defensiva del tirador de la izquierda \\
\hline & INICI_DEF_D & Acción inicial defensiva del tirador de la derecha \\
\hline & INICI_COF_I & Acción inicial contraofensiva del tirador de la izquierda \\
\hline & INICI_COF_D & Acción inicial contraofensiva del tirador de la derecha \\
\hline \multirow{6}{*}{ ACCIÓN FINAL } & FI_OF_I & Acción final ofensiva del tirador de la izquierda \\
\hline & FI_OF_D & Acción final ofensiva del tirador de la derecha \\
\hline & FI_DEF_I & Acción final defensiva del tirador de la izquierda \\
\hline & FI_DEF_D & Acción final defensiva del tirador de la derecha \\
\hline & FI_COF_I & Acción final contraofensiva del tirador de la izquierda \\
\hline & FI_COF_D & Acción final contraofensiva del tirador de la derecha \\
\hline \multirow{3}{*}{ TOCADO } & TOCA_I & Toca el tirador de la izquierda \\
\hline & TOCA_D & Toca el tirador de la derecha \\
\hline & DOBLE & Hay tocado doble \\
\hline
\end{tabular}

Instrumento de registro

Las imágenes fueron codificadas y registradas a través del software libre LINCE v. 1.1 (Gabin, Camerino, Anguera y Castañer, 2012), un programa informático interactivo multimedia, que es compatible con otros programas y plataformas de análisis (Hernández-Mendo, et al., 2014), que permitió visualizar en el mismo monitor del ordenador, la grabación digital de los asaltos y el registro correspondiente al instrumento observacional construido.

\section{Procedimiento}

Se analizaron las imágenes obtenidas del canal YouTube de la Fédération Internationale d'Escrime (FIE Fencing Channel) y de algún vídeo de la televisión venezolana (Televisora Venezolana Social - Tves), correspondientes a los octavos de final y fase final del Campeonato del Mundo de espada de 2013.

El programa informático LINCE permitió registrar todas las co-ocurrencias acontecidas en cada uno de los 14 asaltos analizados, obteniendo un total de 1005 configuraciones (filas de la matriz de códigos en el registro), que se corresponden con cada voz de "halte" (alto) o "allez" (adelante) del árbitro, y con aquellas frases de armas en que el observador consideró que se produjo una acción que entrańaba suficiente intencionalidad para lograr un tocado por parte de alguno de los tiradores. Entendemos por frase de armas el encadenamiento de acciones ofensivas, defensivas y contraofensivas que efectúan los tiradores a lo largo del asalto, en las que el objetivo principal es la consecución del tocado.

Todas las ocurrencias registradas a través de LINCE fueron exportadas al programa Microsoft $\mathrm{O}^{\oplus}$ Office Excel $^{\circledR} 2013$ para ser tratadas y recodificar algunas de las variables cualitativas, y así obtener una matriz con los valores cuantitativos de resumen correspondientes a los 14 asaltos analizados, y a Theme para la detección de T-Patterns. Con esta matriz se realizó un análisis temporal de los asaltos, que contempló el tiempo total de asalto (tiempo transcurrido desde el "allez" inicial que da el árbitro, hasta la finalización del asalto), el tiempo efectivo de combate (suma de segundos que transcurre entre cada "allez" y cada "halte"), el tiempo total de pausa (suma del tiempo de pausa del período, más el tiempo de pausa entre períodos), el tiempo de pausa del período (tiempo que transcurre entre cada "halte" y cada "allez" del período), el tiempo de pausa entre períodos (tiempo transcurrido entre el final de un período y el inicio del siguiente), el tiempo medio por cada "halte" (tiempo medio que tarda en reanudarse un asalto después de un "halte") y el tiempo medio por cada "allez" (tiempo medio transcurrido desde que el árbitro dice "allez" hasta que dice "halte").

Se realizó un análisis asimétrico a través del instrumento observacional, que permitió diferenciar entre las acciones efectuadas por el tirador situado a la izquierda de las imágenes, de las acciones del tirador de la derecha. Esta diferenciación permite el análisis de las interacciones entre ambos deportistas así como la determinación de la secuencialidad en el combate. Para la identificación de las frases de armas más utilizadas en el global de los combates, así como sus regularidades, se optó por eliminar la lateralidad, de izquierda (I) y derecha (D), mediante un análisis en simetría (A, B), recodificando todas las acciones, de manera que el tirador que realizaba la primera acción era identificado como tirador " $A$ ", independientemente de si era el tirador de la izquierda o el de la derecha; su rival era el tirador "B".

El programa informático utilizado para la identificación de T-Patterns fue el Theme v.6 edu (Magnusson, 2005, 2006).

\section{Control de la calidad del dato}

La calidad del dato (Blanco-Villaseñor y Anguera, 2000) fue determinada a través de los procesos de validez y fiabilidad. La validez de constructo se garantizó mediante la consistencia y robustez conceptual, extraída del marco teórico de la esgrima y de la valoración crítica del instrumento observacional ESGRIMOBS, mediante un panel de expertos compuesto por 17 especialistas de esgrima (maestros de armas) que, con un acuerdo del $94 \%$ en el conjunto del instrumento y con un rango del $88 \%$ al 100\%, para cada una de las categorías individualmente, validaron el instrumento. La validación se efectuó mediante un cuestionario virtual (formulario Google) en el que los expertos debían manifestar si estaban de acuerdo o no con la categorización de cada criterio, y en el que podían realizar observaciones para mejorar el instrumento.

Se implementó un período de entrenamiento y formación 
de los observadores en la aplicación del instrumento de registro, con tres expertos en esgrima que establecieron los criterios necesarios para conseguir la concordancia consensuada (Anguera, 1990) en distintos visionados. La fiabilidad en la observación se determinó mediante la concordancia interobservador en 45 acciones de 3 asaltos, con valores superiores a 0,71 en el coeficiente Kappa (Cohen, 1968) en el contraste de los observadores del estudio. El grado de concordancia intraobservador determinó un valor de 0,74 (Kappa de Cohen) en el análisis de 38 acciones en 3 combates. Para este análisis se utilizó el programa SDIS-GSEQ para Windows 4.1.3 (Bakeman y Quera, 2007).

\section{Resultados}

\section{Análisis descriptivos e inferenciales}

De acuerdo al proceso indicado en la metodología del estudio, se codificó un total de 1005 configuraciones en el conjunto de los 16 tiradores de la muestra. Del análisis de la distribución de las frecuencias verificamos que existen diferencias significativas $(p<0,05)$ entre el número de acciones, así como en su eficacia, entre los diferentes períodos de los asaltos (test de $\chi 2$ ). Se transformaron registros cualitativos en variables cuantitativas que resumían las frecuencias observadas y sus estructuras temporales, mediante la media aritmética, desviación estándar $( \pm \mathrm{DE})$, la distribución porcentual y el rango de valores para cada período de los asaltos. Para el análisis de las diferencias entre períodos se llevó a cabo un análisis de varianza (ANOVA). Para cada una de las variables de análisis se comprobó su distribución normal a través de la prueba Shapiro-Wilks $(\mathrm{n}<30)$ y la homogeneidad de varianzas mediante la prueba de Levene. En caso de incumplimiento del supuesto de normalidad y homoscedasticidad, se comprobaron las diferencias entre períodos a través del test $\mathrm{H}$ de Kruskal-Wallis. En estos casos, la comparación por pares se realizó mediante la prueba U de Mann-Whitney con corrección de Bonferroni. El nivel de confianza preestablecido para los contrastes fue $95 \%$ y la probabilidad de error $p<0,05$ (contraste significativo). Para estos cálculos se utilizó el programa PASW Statistics para Windows (v.18, SPSS Inc., Chicago, IL), que es una versión reducida y libre del programa SPSS.

En el conjunto de los 14 asaltos del Campeonato del Mundo de espada masculina de categoría absoluta, el $43,1 \%( \pm 13,3)$ del total de acciones realizadas por los tiradores fueron eficaces, es decir, acabaron en tocado. En la tabla 2 puede apreciarse como existe una tendencia a incrementarse la eficacia de las acciones a medida que avanza el asalto. En las dos únicas prórrogas que hubo en los 14 asaltos, en una se finalizó a los 5,3 s, con una eficacia del $100 \%$ (sólo hubo una acción, y logró el tocado), y en la otra se disputó el minuto entero (al haberse llegado a la prórroga por falta de combatividad), y fueron eficaces el $42,9 \%$ de las acciones efectuadas.

Tabla 2. Eficacia de las acciones según el período en los asaltos de espada (desde octavos de final hasta la final) del Campeonato del Mundo absoluto de 2013.

\begin{tabular}{|c|c|c|c|c|c|c|}
\hline & \multicolumn{2}{|c|}{ ler periodo } & \multicolumn{2}{|c|}{$2^{\circ}$ periodo } & \multicolumn{2}{|c|}{ 3er periodo } \\
\hline & Totales & Eficaces & Totales & Eficaces & Totales & Eficaces \\
\hline Frases de armas (n) & $16,6 \pm 6,2$ & $6,3 \pm 3,7$ & $18,6 \pm 5,8$ & $7,2 \pm 2,7$ & $15,5 \pm 10,2$ & $7,2 \pm 4,9$ \\
\hline Eficacia de las frases de armas (\%) & \multicolumn{2}{|c|}{$35,8 \pm 18,9$} & \multicolumn{2}{|c|}{$42,3 \pm 19,0$} & \multicolumn{2}{|c|}{$47,8 \pm 25,8$} \\
\hline
\end{tabular}

La tabla 3 muestra una descripción detallada de la distribución temporal de los asaltos, período a período, observándose que, el tiempo medio efectivo de combate representa el $45 \%$ del tiempo total medio de todos los asaltos, y el tiempo total de pausa el $55 \%$.

En general, a medida que avanza el asalto, el tiempo efectivo de combate disminuye y se alargan los tiempos de pausa de los períodos. El tiempo por "allez" se sitúa en un rango entre los 0,9 y los $76,8 \mathrm{~s}$, siendo $17,1 \mathrm{~s}( \pm 4,1)$ el tiempo medio por "allez" y 17,3 s $( \pm 3,5)$ el tiempo medio por "halte".

No existen diferencias significativas entre los diferentes tiempos analizados de los períodos, a excepción de las diferencias existentes en cuanto al tiempo medio por "allez", entre el primer y tercer período de los asaltos $(p<0,05)$.

Tabla 3. Descripción cronométrica de los asaltos de espada (desde octavos de final hasta la final) del Campeonato del Mundo absoluto de 2013.

\begin{tabular}{|c|c|c|c|}
\hline & ler periodo & $2^{\circ}$ periodo & 3er periodo \\
\hline Asaltos (n) & 14 & 14 & 10 \\
\hline Tiempo total de asalto (s) & $343,2 \pm 113,0$ & $362,1 \pm 94,5$ & $274,6 \pm 163,6$ \\
\hline Tiempo efectivo de combate (s) & $164,0 \pm 39,4$ & $157,4 \pm 35,8$ & $116,0 \pm 58,7$ \\
\hline Tiempo total de pausa (s) & $179,2 \pm 76,7$ & $204,6 \pm 76,7$ & $158,6 \pm 106,9$ \\
\hline Tiempo de pausa del periodo (s) & $117,7 \pm 55,1$ & $145,5 \pm 81,5$ & $190,9+78,8$ \\
\hline Tiempo de pausa entre periodos (s) & $70,0 \pm 32,9$ & $82,8 \pm 29,3$ & $29,7 \pm 6,0$ \\
\hline Frases de armas (n) & $15,9 \pm 6,1$ & $17,9 \pm 5,6$ & $14,8 \pm 9,7$ \\
\hline "Halte" (n) & $7,9 \pm 4,0$ & $9,5+3,1$ & $8,7 \pm 5,4$ \\
\hline Tiempo medio pora "halte" (s) & $16,8 \pm 6,0$ & $16,5 \pm 3,7$ & $19,9 \pm 5,2$ \\
\hline Tiempo medio por "allez" (s) & $26,6 \pm 13,1 \#$ & $19,2 \pm 10,1$ & $18,2 \pm 15,7$ * \\
\hline Acciones por frase de armas (n) & $2,2 \pm 0,3$ & $2,3 \pm 0,2$ & $2,5 \pm 0,4$ \\
\hline
\end{tabular}

\section{Detección de T-Patterns}

En la tabla 4 queda resumido el resultado de la detección de T-Patterns en los 14 asaltos. Se detectaron un total de $72 T$ Patterns (tabla 4) con una distribución irregular en los asaltos. Del total de 223 distintas frases de armas registradas (distintas combinaciones de co-ocurrencias), 38 formaron parte de alguno de los T-Patterns, apareciendo, en el conjunto de los asaltos en un total de 188 ocurrencias. El máximo número de T-Patterns se detectaron en el asalto $1(\mathrm{n}=19)$, mientras que en el asalto 8 no se detectó ninguno. En la tabla 4 se detallan las configuraciones que componen los 20 T-patterns más comunes. 
Tabla 4. Distribución de las configuraciones de los T-Patterns detectados y más comunes en los asaltos de la especialidad de espada masculina de la fase final del Campeonato del Mundo de Esgrima.

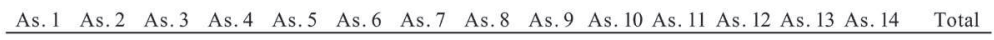

\begin{tabular}{llllllllllllllll} 
Total T-Patterns & 19 & 2 & 2 & 4 & 3 & 4 & 14 & 0 & 9 & 1 & 1 & 1 & 10 & 2 & 72 \\
\hline T-Patterns & 52 & 4 & 5 & 8 & 7 & 8 & 47 & 0 & 25 & 2 & 2 & 2 & 22 & 4 & 188 \\
Frases de armas en T-Patterns & & & &
\end{tabular}

Principales frases de armas no efectivas en los T-Pattern (entre los 20 más frecuentes)

pres_d,prep_d,inici_of_d,fi_def_i,

pres_i,nprep,inici_of_i,fi_def_d,

pres_i,prep_d,inici_of_i,fi_def_d,

npres,prep_d,inici_of_d,fi_def $\mathrm{i}$,

npres,nprep,inici_of_i,fi_def_d,

pres_i,prep_i,inici_of_i,fi_def_d,

pres_d,prep_i,inici_of_d,fi_def_i,

pres d,prep d,inici of i,fi def

pres_i,prep_i,inici_of_d,fi_def_i,

npres,prep_d,inici_def_i,

npres,prep_i,inici_of_d,fi_def_i,

npres,prep_i,inici_of_i,fi_def_d,

npres,prep d,inici of $\mathrm{i}, \overline{\mathrm{fi}} \mathrm{de} \overline{\mathrm{d}}$

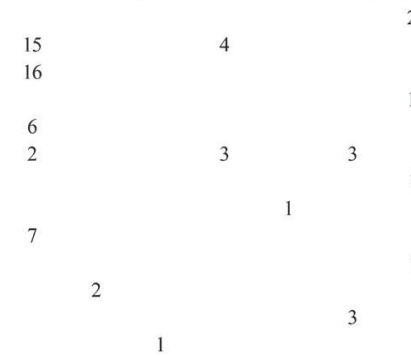

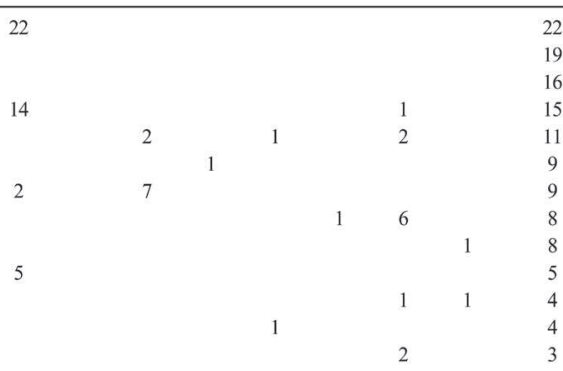

Principales frases de armas efectivas en los T-Pattern (entre los 20 más frecuentes)

pres_i,prep_i,inici_of_i,fi_cof_d,toca_i 3

pres d.prep d,inici of $i$,fi cof d,toca $i$

pres d.prep d,inici of d,fi cof i,toca $i$

pres d,prep d,inici of d,fi cof i,doble

pres_i,prep_i,inici_of_i,fi_cof_d,doble

pres_d,prep_d,inici_of_i,fi_def_d,toca_d

npres,prep d,inici of i,fi def d,toca d

La tabla 5 nos muestra las frases de armas más frecuentes en los principales T-patterns detectados en los asaltos, detallándose la aparición de las 20 más comunes. La numeración se corresponde al orden de aparición de los patrones (T-patterns $1,2,3 \ldots 19)$ y a la distribución secuencial de sus acciones (1a,

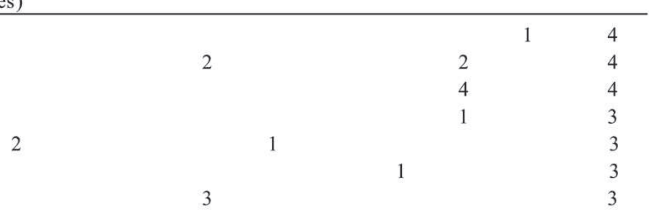

1b... 1d; 2a, 2b...). En la figura 1 se describe la nomenclatura utilizada para facilitar la interpretación de los distintos patrones de la tabla 5 según las frases de armas realizadas y su secuencialidad en los distintos asaltos.

Tabla 5. Ubicación de las configuraciones que componen los T-Patterns detectados en asaltos de espada masculina de la fase final del Campeonato del Mundo de Esgrima.

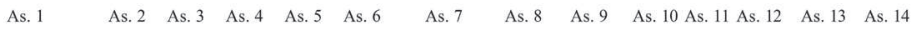

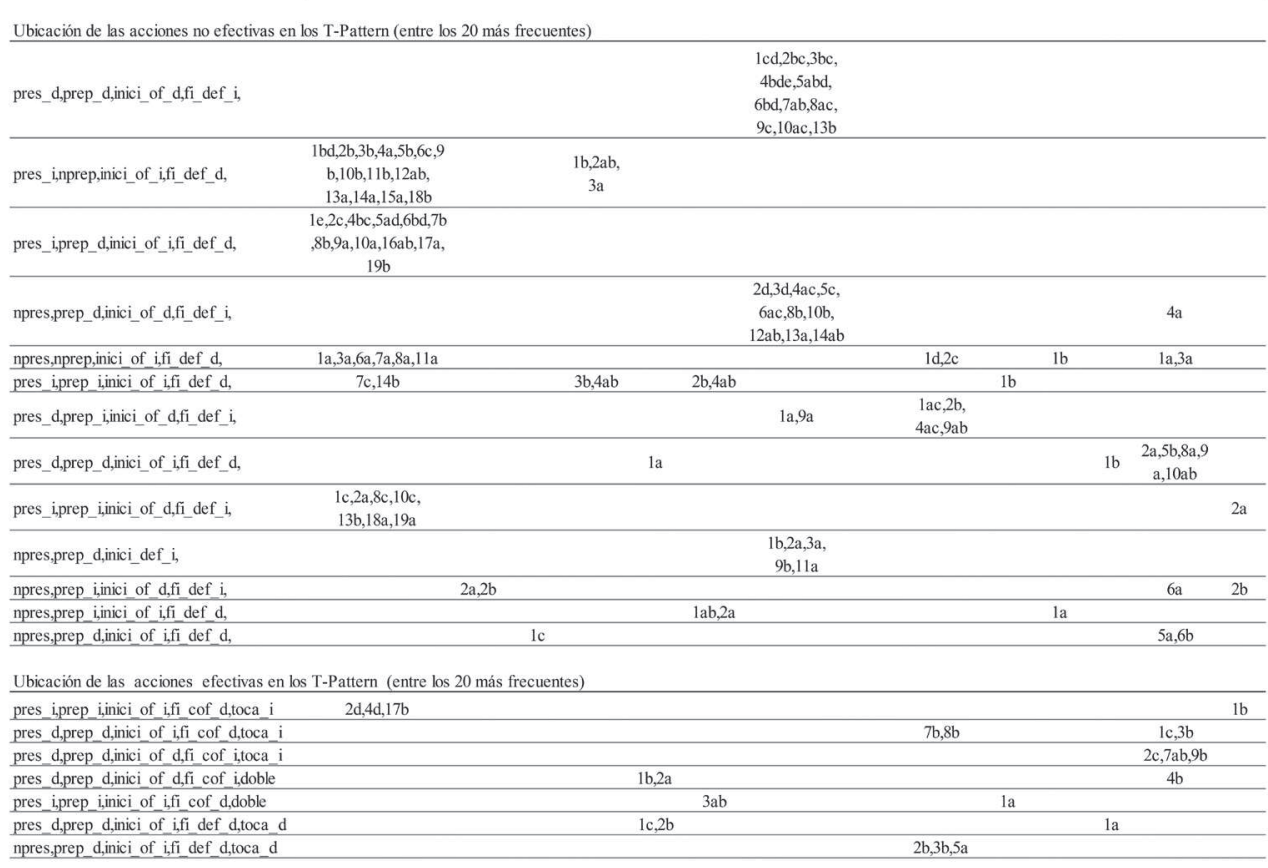


Figura 1. Dendogramas de distribución de las configuraciones de los T-patterns más comunes.

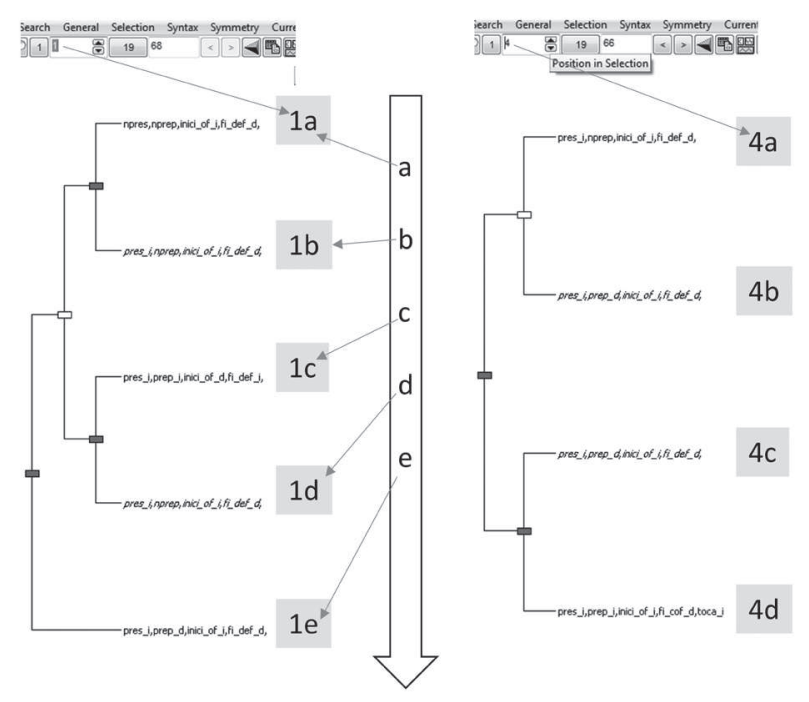

En la figura 2 se han representado los ocho primeros patrones que consiguen acciones eficaces sobre el marcador en el análisis simétrico de los registros.

Figura 2. Dendogramas que representan las configuraciones eficaces de los T-patterns de las acciones ( $\mathrm{n}=620)$ analizadas en simetría $(\mathrm{A}, \mathrm{B})$, en asaltos del Campeonato del Mundo de espada.

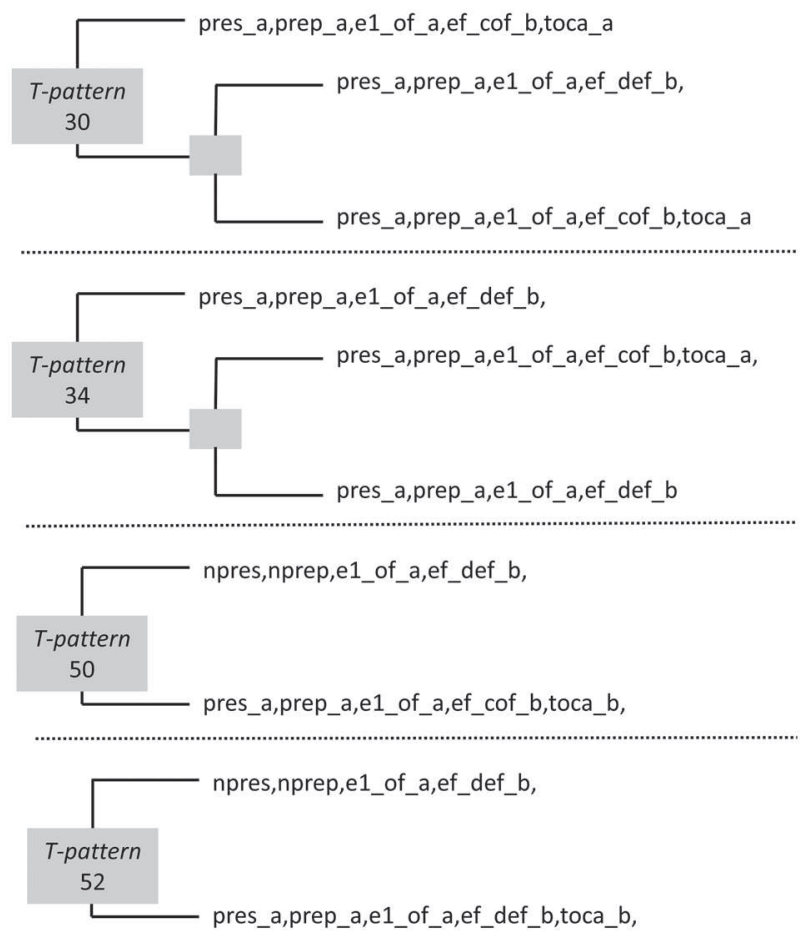

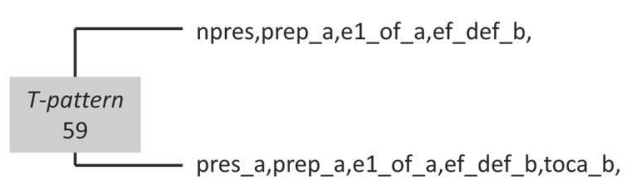
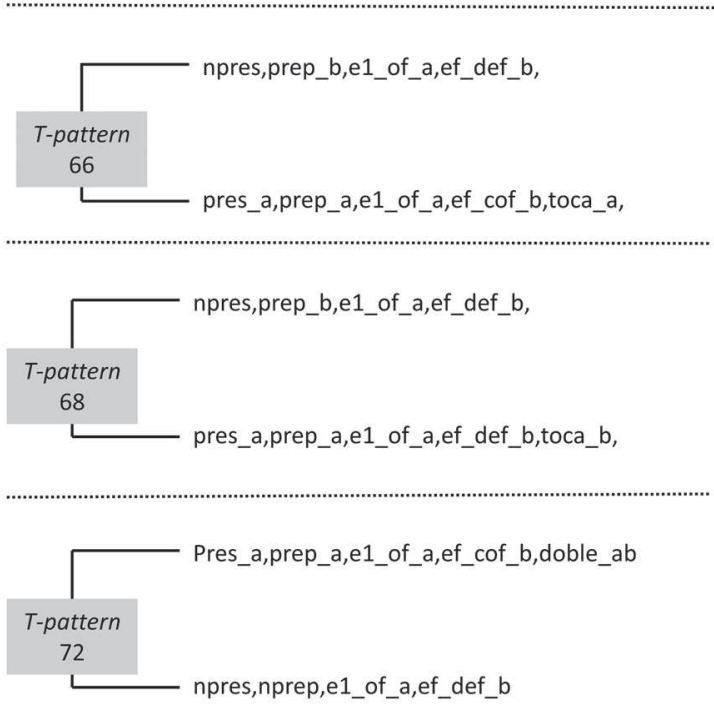

obtención de acciones eficaces fue aquel en el que el tirador que inicia la acción ofensiva (e1_of_a) es quien presiona a su rival (pres_a), realiza la última acción preparatoria (prep_a), y, ante una acción final contraofensiva del rival (ef_cof_b), termina ganando el tocado (toca_a). 


\section{Discusión}

Los resultados de este estudio nos mostraron que el instrumento observacional que construimos (ESGRIMOBS) nos permitió contextualizar lo sucedido en un asalto, analizando su estructura temporal y, por primera vez en la literatura, las frases de armas acontecidas durante el asalto, identificando estructuras regulares de conducta.

Los resultados obtenidos indican que la eficacia de las acciones presenta una tendencia a incrementarse a medida que avanza el asalto, lo cual no coincide con un estudio anterior (Iglesias, et al., 2010), también en espada masculina de alto nivel, en el que las acciones del tercer período eran las menos eficaces. Ambos estudios coinciden en señalar que no se aprecia una relación estadísticamente significativa que condicione la eficacia de las acciones al período en que se ejecutan.

En la tabla 2 también se aprecia que el número medio de acciones que se realizan en cada período de un asalto es bastante similar, lo que en un principio podría sorprender si tenemos en cuenta la progresiva finalización de los asaltos al llegar a los 15 tocados o al agotarse el tiempo del asalto. Contrastando la estadística con los procesos observacionales de los videos se comprobó que en el primer período de dos de los asaltos, el árbitro consideró que no había combatividad, dando paso al siguiente período, y que cuatro de los asaltos analizados finalizaron antes de concluir los 3 minutos reglamentarios del segundo período, al llegar el marcador a los 15 tocados. Por tanto, si bien es cierto que a priori cabe pensar que los dos primeros períodos se disputan de manera completa, hay ciertas circunstancias que pueden hacer disminuir el tiempo efectivo de combate.

La explicación que damos al incremento de la eficacia de las acciones a medida que avanza el asalto, y al volumen similar de acciones por período, a pesar de que no se llegue a disputar la totalidad del tercer tiempo -como sucedió en el 60\% de los asaltos con tercer tiempo disputado- lo atribuimos a la presión ambiental (Gasset y Iglesias, 2010). El ir por debajo en el marcador, cuando el tiempo del combate está llegando a su fin, obliga al tirador a tomar riesgos que dan lugar a la realización de acciones más definitivas, y que se traducen en la consecución de un tocado por parte de uno de los tiradores, incrementando la eficacia de las acciones en el marcador.

En relación a la caracterización de las acciones temporales en la esgrima, encontramos estudios que realizaron un cálculo de la duración de los movimientos de alta intensidad en los asaltos (Wylde, et al., 2013), otros que analizaron el tiempo total medio de asalto, el tiempo total de todos los asaltos y el tiempo total de una competición (Iglesias y Rodríguez, 1995), también los hay que cuantificaron el tiempo total de asalto y el tiempo efectivo de combate para asaltos a 5 y 10 tocados (Lavoie, et al., 1984), incluso existe algún trabajo en el que se cuantificó la actividad temporal del ganador de una com- petición, especificando el tiempo que duró la competición, el tiempo de descanso entre asaltos, el tiempo total de asalto, el tiempo efectivo de combate y el tiempo total de pausa (Roi, et al., 2008).

Los resultados del estudio cronométrico de los asaltos de este trabajo (tabla 3) muestran que el tiempo medio efectivo de combate representa el $45 \%$ del tiempo total medio de todos los asaltos, y el tiempo total de pausa el 55\%, resultados que se asemejan a los de Pittaluga (Pittaluga y Roi, 1999), en el que el tiempo medio efectivo de combate representaba un $42 \%$ y el tiempo total de pausa el $58 \%$.

El tiempo medio por "allez" obtenido es de $17,1 \mathrm{~s}( \pm 4,1)$, mientras que el tiempo medio por "halte" de 17,4 s $( \pm 3,5)$. Estos resultados son parecidos a los obtenidos por Pittaluga, et al., (1999) en cuanto al tiempo medio por "halte", cuantificado en $18,2 \mathrm{~s}( \pm 12,3)$, pero no en lo referente al tiempo medio por "allez", de un valor de 12,7 s $( \pm 7,6)$.

En la tabla 3 se puede apreciar como el avance del asalto va acompañado por un incremento de los tiempos de pausa de los períodos y una disminución de los tiempos efectivos de combate. El hecho que a medida que el asalto avanza sea mayor la presión temporal (Iglesias, et al., 2010) también puede influir en la ampliación por parte de los tiradores de los tiempos de pausa, con el objetivo de disponer de más tiempo de reflexión, entre tocados, para replantear su estrategia. De los asaltos analizados, el 29\% de ellos finalizó antes de concluir el tiempo reglamentario del segundo período, y el 60\% de los que llegaron al tercer período finalizaron sin agotar los 3 minutos.

En la literatura de esgrima no hay demasiados trabajos sobre la toma de decisiones de los deportistas, como sí sucede en otros deportes (Refoyo, Domínguez, Sampedro y del Campo, 2009; Sáez-Gallego, Vila-Maldonado, Abellán y Contreras, 2013), aunque sí se han realizado interesantes aportaciones sobre las habilidades psicológicas de los esgrimistas (RecheGarcía, Cepero-González y Rojas-Ruiz, 2010, 2013). Por dicha razón, la aportación que hace este estudio, analizando, por primera vez, las frases de armas de la esgrima, puede ser de gran valor para el análisis táctico de los asaltos.

En el registro de las conductas realizadas por los tiradores se observó intercambios de entre 2 a 8 distintas acciones dentro de una misma frase de armas (secuencias continuadas de ataques, paradas, respuestas, contraataques...) por parte de los dos tiradores. Para simplificar el análisis se acordó utilizar la última acción, de cada frase de armas, anterior a la finalización de la acción. El último criterio registrado fue el carácter del tocado, pudiendo ser un tocado simple, para alguno de los dos rivales, o bien un tocado doble. En el análisis secuencial la finalización de la acción sin tocado se mostró como ausencia de esta ocurrencia.

La detección de T-Patterns (tabla 4), con un requerimiento de 2 ocurrencias como mínimo, y un nivel de significación de 
0,05, mostró que las frases de armas más utilizadas son aquellas en las que existe ausencia de tocado, es decir, acciones en las que la categoría que cierra la co-ocurrencia es una acción que genera una ausencia de tocado. Esta acción final se produjo con una diversificación de tipos de frases de armas, con condicionantes de presión y preparación diversos por parte de los dos rivales, así como de inicio de la primera acción. Observamos cómo, en el análisis de las frases de armas (tabla 4), no apareció una co-ocurrencia que derivase en la consecución de tocado entre las más frecuentes. La frase de armas que finalizó en tocado con mayor regularidad en la detección de T-Patterns fue aquella en la que el tirador de la izquierda realizaba la presión, la preparación última y la acción ofensiva, consiguiendo el tocado ante la reacción contraofensiva del adversario (derecha). Si tomamos como ejemplo el asalto uno, podemos comprobar que esta regularidad está presente en tres T-Patterns, lo que indica un patrón de conducta muy característico del tirador de la izquierda.

Para profundizar en el análisis de las regularidades de cada uno de los deportistas fue necesario abordar el análisis asimétrico en cada uno de los combates mediante los T-Patterns detectados. La tabla 5 presenta un resumen esquemático de la presencia de las 20 frases de armas más frecuentes en cada uno de los patrones detectados en los combates del estudio. Se puede apreciar que en el asalto 1 existe una gran cantidad de regularidades, repitiéndose estructuras en las que prima una presión del tirador de la izquierda (pres_i) en casi todo el combate, con alternancia entre los dos deportistas en la ejecución de la preparación (prep_i y prep_d) y una predominancia en la primera acción ofensiva del tirador de la izquierda (inici_of_i); cuando la reacción del rival fue defensiva (fi_def_d) nadie consiguió el tocado, pero cuando el rival reaccionó con una acción contraofensiva (fi_cof_d) el atacante (izquierda) ganó el tocado mayoritariamente (toca_i). Este tipo de análisis podría contribuir a detectar, en el conjunto de acciones de un combate, regularidades entre los tiradores que permitieran al maestro de esgrima mejorar el conocimiento de sus deportistas, y de sus rivales, optimizando sus procesos de preparación estratégica.

Con el objetivo de valorar las acciones más utilizadas, en el conjunto de los combates, se realizó una recodificación de los registros observados. El análisis asalto a asalto requirió de un análisis asimétrico, diferenciando al tirador de la derecha en relación al de la izquierda para ver qué acciones ejecutaban cada uno de los rivales. En el análisis simétrico eliminamos la lateralidad $(\mathrm{I}, \mathrm{D})$ para convertirla en simetría $(\mathrm{A}, \mathrm{B})$ dónde todas las acciones las hemos recodificado determinando como tirador "A" aquel que realizaba la primera acción. En la figura 2 se pueden apreciar los T-Patterns que derivaron en una ac- ción efectiva, observando que de los 98 T-Patterns detectados, no aparecía una regularidad que generase acción efectiva hasta el patrón número 30. Se ha descrito en los resultados como en este patrón (pres_a,prep_a,el_of_a,ef_cof_b,toca_a) la acción ofensiva del tirador $\mathrm{A}$ vino precedida por la presión $\mathrm{y}$ preparación del mismo tirador, y ante la contraofensiva de B, el atacante (A) ganó el tocado. Se puede apreciar que esta acción simétrica, es coincidente con la asimétrica (I,D) descrita anteriormente, y que se constituye en una de las principales regularidades en estos combates.

En conclusión, este estudio muestra que se dispone de una herramienta (ESGRIMOBS) de gran potencial para lograr la caracterización técnico-táctica de los asaltos de esgrima, al permitir realizar un análisis cronométrico de los asaltos, cuantificar el volumen de acciones y su eficacia, analizar las frases de armas que se producen durante los asaltos y facilitar la identificación los patrones temporales de conducta que se dan en la interacción entre los esgrimistas.

\section{Aplicaciones prácticas}

El análisis de la estructura temporal de los distintos asaltos debe contribuir a mejorar el conocimiento de las dinámicas temporales de esta especialidad, incorporando entrenamientos de asalto orientados a situaciones de limitación del tiempo disponible ante marcadores diversos. La preparación física y técnica de los deportistas debe adecuarse a estos referentes temporales, valorando los tiempos de trabajo y reposo entre ejecuciones técnicas. El análisis de los T-Patterns puede constituir una valiosa herramienta en el futuro de la optimización de la preparación estratégica de la esgrima, mediante el estudio de rivales y el análisis de las regularidades de los deportistas de cada maestro de esgrima.

Agradecimientos: Este trabajo ha sido posible gracias al apoyo del Institut Nacional d'Educació Física de Catalunya (INEFC Barcelona), a la Federació Catalana d'Esgrima y a los maestros de esgrima que han contribuido en las distintas fases de este estudio.

El estudio ha contado con recursos del Institut Nacional d'Educació Física de Catalunya (INEFC Barcelona) y AGAUR (PINEFC PRE 2730/2012, SGR 971 y SGR 1665), y forma parte del proyecto «Observación de la interacción en deporte y actividad física: avances técnicos y metodológicos en registros automatizados cualitativos-cuantitativos", subvencionado por la Secretaría de Estado de Investigación, Desarrollo e Innovación del Ministerio de Economía y Competitividad (DEP2012-32124). 


\section{Referencias}

1. Anguera, M. T. (1990). Metodología observacional. In J.Arnau, M. T. Anguera y J. Gómez, (Eds.), Metodología de la investigación en ciencias del comportamiento (pp. 125-238). Murcia: Universidad de Murcia.

2. Anguera, M. T., Blanco-Villaseñor, A., Hernández-Mendo, A. y Losada, J. L. (2011). Diseńos observacionales: ajuste y aplicacion en psicologia del deporte. Cuadernos de Psicologia del Deporte, 11(2), 63-76.

3. Aquili, A., Tancredi, V., Triossi, T., Sanctis, D. D., Padua, E., D’Arcangelo, G. y Melchiorri, G. (2013). Performance Analysis in Saber. The Journal of Strength \& Conditioning Research, 27(3), 624-630.

4. Bakeman, R. y Quera, V. (2007). Software SDIS-GSEQ, versión 4.1.3.

5. Blanco-Villaseñor, A. y Anguera, M.T. (2000). Evaluación de la calidad en el registro del comportamiento: Aplicación a deportes de equipo. En E. Oñate, F. García-Sicilia y L. Ramallo (Eds.), Métodos numéricos en Ciencias Sociales (pp. 30-48). Barcelona: Centro Internacional de Métodos Numéricos en Ingeniería (CIMNE).

6. Bonifazi, M., Rossi, S. y Vannoni, B. (2009). Incidence of fencing injuries. Analysis of a survey of elite fencers. Medicina Dello Sport, 62(2), 177-192.

7. Borrie, A., Jonsson, G. K. y Magnusson, M. S. (2002). Temporal pattern analysis and its applicability in sport: an explanation and exemplar data. Journal of Sports Sciences, 20(10), 845-852.

8. Bressan, A. y Ranzani, P. (1998). La valutazione funzionale degli arti inferiori nella scherma: Valutazione funzionale riguardante alcune esercitazioni di potenza muscolare degli arti inferiori nella scherma. Sds/Rivista di Cultura Sportiva - CONI, 17, 104-119.

9. Camerino, O., Prieto, I., Lapresa, D., Gutiérrez-Santiago, A. y Hileno, R. (2014). Detección de T-patterns en la observación de deportes de combate. Revista de Psicologia del Deporte, 23(1), 147-155.

10. Camerino, O., Chaverri, J., Anguera, M. T. y Jonsson, G. K. (2012). Dynamics of the game in soccer: Detection of T-patterns. European Journal of Sport Science, 12(3), 216-224.

11. Cohen, J. (1968). Weighted kappa: Nominal scale agreement provision for scaled disagreement or partial credit. Psychological Bulletin, 70(4), 213.

12. Fernández, J., Camerino, O., Anguera, M. T. y Jonsson, G. K. (2009). Identifying and analyzing the construction and effectiveness of offensive plays in basketball by using systematic observation. Behavior Research Methods, 41(3), 719-730.

13. FIE (2014). Reglamentos. Recuperado el 20 de junio de 2014 de http:// www.fie.ch/Fencing/Rules.aspx

14. Gabin, B., Camerino, O., Anguera, M. T. y Castañer, M. (2012). Lince: multiplatform sport analysis software. Procedia-Social and Behavioral Sciences, 46, 4692-4694.

15. Gasset, A. y Iglesias, X. (2010). Caracterización de la presión temporal como factor ambiental de la conducta humana en situación de conflicto. El caso de la esgrima de espada. In $V$ Congreso Internacional de Educación Física: Barcelona (pp. 131-137).

16. Gholipour, M., Tabrizi, A. y Farahmand, F. (2008). Kinematics analysis of lunge fencing using stereophotogrametry. World Journal of Sport Sciences, 1(1), 32-37.

17. Gutiérrez-Dávila, M., Zingsem, C., Gutiérrez-Cruz, C., Giles, F. J. y Rojas, F. J. (2014). Effect of Uncertainty during the Lunge in Fencing. Journal of Sports Science \&Medicine, 13(1), 66.

18. Gutiérrez-Santiago, A., Prieto, I., Camerino, O. y Anguera, M.T. (2011). The temporal structure of judo bouts in visually impaired men and women. Journal of Sports Science, 29(13), 1443-1451.

19. Harmenberg, J., Ceci, R., Barvestad, P., Hjerpe, K. y Nystrom, J. (1991). Comparison of different tests of fencing performance. International Journal of Sports Medicine, 12(6), 573-576.

20. Harriss, D. y Atkinson, G. (2011). Update-ethical standards in sport and exercise science research. International Journal of Sports Medicine, 32(11), 819-821.

21. Hernández-Mendo, A., Castellano, J., Camerino, O., Jonsson, G., Blanco-Villaseñor, A., Lopes, A. y Anguera, M. T. (2014). Programas informáticos de registro, control de calidad del dato, y análisis de datos. Revista de Psicología del Deporte, 23(1), 111-121.

22. Iglesias, X. (1997). Valoració funcional especifica en l'esgrima. Doctoral dissertation, Universitat de Barcelona, Barcelona, Spain.

23. Iglesias, X., Gasset, A., González, C. y Anguera, M. T. (2010). Interacción competitiva y presión ambiental en deportes de combate: Aplicación de la metodología observacional. Revista Iberoamericana de Psicología del Ejercicio y el Deporte, 5(2), 267-282.

24. Iglesias, X., González, C., Cortés, A., Tarragó, R. y García, J. J. (2008). Influence of the first hit in the final score of an épée bout. In X. Iglesias (Ed.) 1st International Congress on Science and Technology in Fencing: Barcelona (pp. 149-152).

25. Iglesias, X., González, C., Cortés, A., Tarragó, R. y García, J. J. (2008). Variability of technical actions in épée fencing. In X. Iglesias (Ed.) 1st International Congress on Science and Technology in Fencing: Barcelona (pp. 153-156).

26. Iglesias, X. y Rodríguez, F. A. (1995). Caracterización de la frecuencia cardiaca y la lactatemia en esgrimistas durante la competición. Apunts Medicina de l'Esport, 32, 21-32.

27. Jonsson, G. K., Anguera, M. T., Blanco-Villasenor, A., Losada, J. L., Hernández-Mendo, A., Ardá, T., Camerino, O. y Castellano, J. (2006). Hidden patterns of play interaction in soccer using SOF-CODER. Behavior Research Methods, 38(3), 372-381.

28. Lapresa, D., García, J., Arana, J. y Garzón, B. (2011). Análisis de patrones temporales en las rutinas gestuales previas al tiro libre de baloncesto, en la categoría alevín. Revista de Psicología del Deporte, 20(2), 383-400.

29. Lapresa, D., Ibáńez, R., Arana, J., Garzón, B. y Amatria, M. (2011). Spatial and temporal analysis of karate kumite moves: Comparative study of the senior and 12-13 year old groups. International Journal of Performance Analysis in Sport, 11(1), 57-70.

30. Lavoie, J., Léger, L., Pitre, R. y Marini, J. (1985). Compétitions d'escrime. Épée. Analyse des durées et distances de déplacement. $M e$ dicine du Sport, 5(59), 279-283.

31. Lavoie, J. M., Léger, L. y Marini, J. F. (1984). Comparaisons anthropométriques et physiologiques de deux niveaux d'escrimeurs compétitifs. La Revue Québécoise de l'Activité Physique, 3(2), 91-95.

32. Lin, F. (2004). Optimal handle angle of the fencing foil for improved performance 1, 2. Perceptual and Motor Skills, 98(3), 920-926.

33. Magnusson, M. S. (1996). Hidden real-time patterns in intra-and inter-individual behavior: Description and detection. European Journal of Psychological Assessment, 12(2), 112-123.

34. Magnusson, M. S. (2000). Discovering hidden time patterns in behavior: T-patterns and their detection. Behavior Research Methods, Instruments \& Computers, 32(1), 93-110.

35. Magnusson, M. S. (2005). Understanding social interaction: Discovering hidden structure with model and algorithms. In L. Anolli, S. Duncan, M. Magnusson y G. Riva (Eds.), The hidden structure of interaction: From Genomics to Culture Patterns (pp. 4-24). Amsterdam: IOS Press.

36. Magnusson, M.S. (2006) Structure and Communication in Interaction. In G. Riva, M.T. Anguera, B.K. Wiederhold y F. Mantovani (Eds.) From Communication to Presence: Cognition, Emotions and Culture towards the Ultimate Communication Experience (pp. 127-146). Amsterdam: IOS Press.

37. Naghavi, H. (2002). A study of traumatic events in junior fencing 
competitions. Medicine \& Science in Sports \& Exercise, 34(5), 56.

38. Pittaluga, I. y Roi, G. S. (1999). Analisi cinematica della prestazione. Proceedings of the Congress "Il modello di prestazione della scherma moderna", Padova.

39. Reche-García, C., Cepero-González, M. y Rojas-Ruiz, F. J. (2010). Efecto de la experiencia deportiva en las habilidades psicológicas de esgrimistas del ranking nacional español. Cuadernos de Psicología del Deporte, 10(2), 33-42.

40. Reche-García, C., Cepero-González, M. y Rojas-Ruiz, F. J. (2013). Consideraciones en el entrenamiento de habilidades psicológicas en esgrima. Cuadernos de Psicología del Deporte, 13(2), 83-88.

41. Refoyo, I, Domínguez, J., Sampedro, J. y del Campo, J. (2009). Análisis de la decisión táctica en el bloqueo directo. Una comparación entre selecciones internacionales absolutas y clubes. Cuadernos de Psicología del Deporte, 9, 63.

42. Roi, G. y Fasci, A. (1988). Survey of Requests for Medical Assistance During Fencing Matches. Italian Journal of Sports Traumatology, 10(1), $55-62$.
43. Roi, G. S. y Bianchedi, D. (2008). The science of fencing: implications for performance and injury prevention. Sports Medicine, 38(6), 465-481.

44. Sáez-Gallego, N., Vila-Maldonado, S., Abellán, J. y Contreras, O.R. (2013). Análisis del comportamiento visual y la toma de decisiones en el bloqueo en voleibol. Cuadernos de Psicología del Deporte, 13(2), 3144.

45. Tsolakis, C. y Vagenas, G. (2010). Anthropometric, Physiological and Performance Characteristics of Elite and Sub-elite Fencers. Journal of Human Kinetics, 23(1), 89-95.

46. Turner, A., Miller, S., Stewart, P., Cree, J., Ingram, R., Dimitriou, L., Moody y Kilduff, L. (2013). Strength and Conditioning for Fencing. Strength \& Conditioning Journal, 35(1), 1-9.

47. Williams, L. R. y Walmsley, A. (2000). Response timing and muscular coordination in fencing: a comparison of elite and novice fencers. Journal of Science and Medicine in Sport, 3(4), 460-475.

48. Wylde, M. J., Tan, F. H. y O’Donoghue, P. G. (2013). A time-motion analysis of elite women's foil fencing. International Journal of Performance Analysis in Sport, 13(2), 365-376. 